\title{
Long-term memory of real-world episodes is independent of recency effects: magic tricks as ecological tasks.
}

\author{
David Bestue ${ }^{1}$, Luis M. Martínez ${ }^{2}$, Alex Gomez-Marin ${ }^{3}$, Miguel A. Gea ${ }^{4}$ and Jordi Camí ${ }^{5}$ \\ ${ }^{1}$ Institut d'Investigacions Biomèdiques August Pi i Sunyer (IDIBAPS), Barcelona, Spain \\ ${ }^{2}$ Visual Analogy Lab, Instituto de Neurociencias (CSIC-UMH), Alicante, Spain \\ ${ }^{3}$ Behavior of Organisms Lab, Instituto de Neurociencias (CSIC-UMH), Alicante, Spain \\ ${ }^{4}$ Professional magician, Madrid, Spain \\ ${ }^{5}$ Universitat Pompeu Fabra (UPF), Barcelona, Spain
}

Correspondence: info@davidbestue.com or jordi.cami@upf.edu

keywords: episodic memory, serial-position effects, magic, cognition.

How episodic memories decay is an unresolved question in cognitive neuroscience. The role of short-term mechanisms regarding the decay of episodic memories is circumscribed to set the maximum recall from which a monotonic decay occurs. However, this sequential view from the short to the long-term is not compulsory, as short-term dependent memory gains (like recency effects when memorizing a list of elements; serial-position effects) may not be translated into long-term memory differences. Moreover, producing memorable events in the laboratory faces important challenges, such as recreating realistic conditions with elevated recall, or avoiding spontaneous retrievals during memory retention (sociocultural hooks). The current study proposes the use of magic to enhance the study of memory. We designed a sequence of magic tricks performed live on stage to evaluate the interaction between memory decay and serial-position effects of those tricks. The audience was asked to freely recall the tricks at four different timepoints: just after the show, 10 days, 1.5 months and 4.5 months. We discovered serial-position differences after the show that were no longer present later on, suggesting that short-term memory gains do not translate into the long-term. Illustrating the power of naturalistic stimuli to study long-term memory while interrogating the interaction between short-term and long-term mechanisms, this work is, to our knowledge, the first scientific study of the memorability of magic tricks.

\footnotetext{
This paper has already been published by Helyon!
}

David Bestue, Luis M. Martínez, Alex Gomez-Marin, Miguel A. Gea, Jordi Camí,

Long-term memory of real-world episodes is independent of recency effects: magic tricks as ecological tasks, Heliyon, Volume 6, Issue 10, 2020, e05260, ISSN 2405-8440,

https://doi.org/10.1016/j.heliyon.2020.e05260.

(http://www.sciencedirect.com/science/article/pii/S2405844020321034) 


\section{Introduction}

The study of episodic memory, the memory of our everyday personal experiences (Tulving, 1972), is challenging when aiming for naturalistic conditions (Chow \& Rissman, 2017; Plancher \& Piolino, 2017) for different reasons. The fact that episodic memories are formed after a single exposure constitute a critical inconvenience in terms of sample size (Davachi \& DuBrow, 2015). Besides, increasing the complexity of naturalistic stimulus complicates memory evaluation: while simple stimuli are easily testable, complex ones are more susceptible to subject variability. The complexity of the stimulus is related to its emotional relevance; it should be high enough to have detailed memory recall long time after but avoiding stressful or traumatic experiences (Gold et al., 2001; McGaugh \& Roozendaal, 2002; Hirst et al., 2009). Finally, memory consolidation is critically affected by how frequently a memory is retrieved (Hebb, 1961; Sara, 2000; Hardt et al., 2009; Alberini, 2011). Episodic memories are easily retrieved when they have multiple associations (Hargreaves et al., 2012). Retrieving part of a memory reinstate the rest of it (Horner et al., 2015), so it is likely that memories with more sociocultural associations are easily spontaneously retrieved. In each of these spontaneous retrievals, the memory can be contaminated, so finding naturalistic events with minimal conscious retrieval is key. Therefore, the ideal stimulus to study episodic memory formation would be a complex one, easily testable, with elevated recall in the long-term, and minimal spontaneous retrieval.
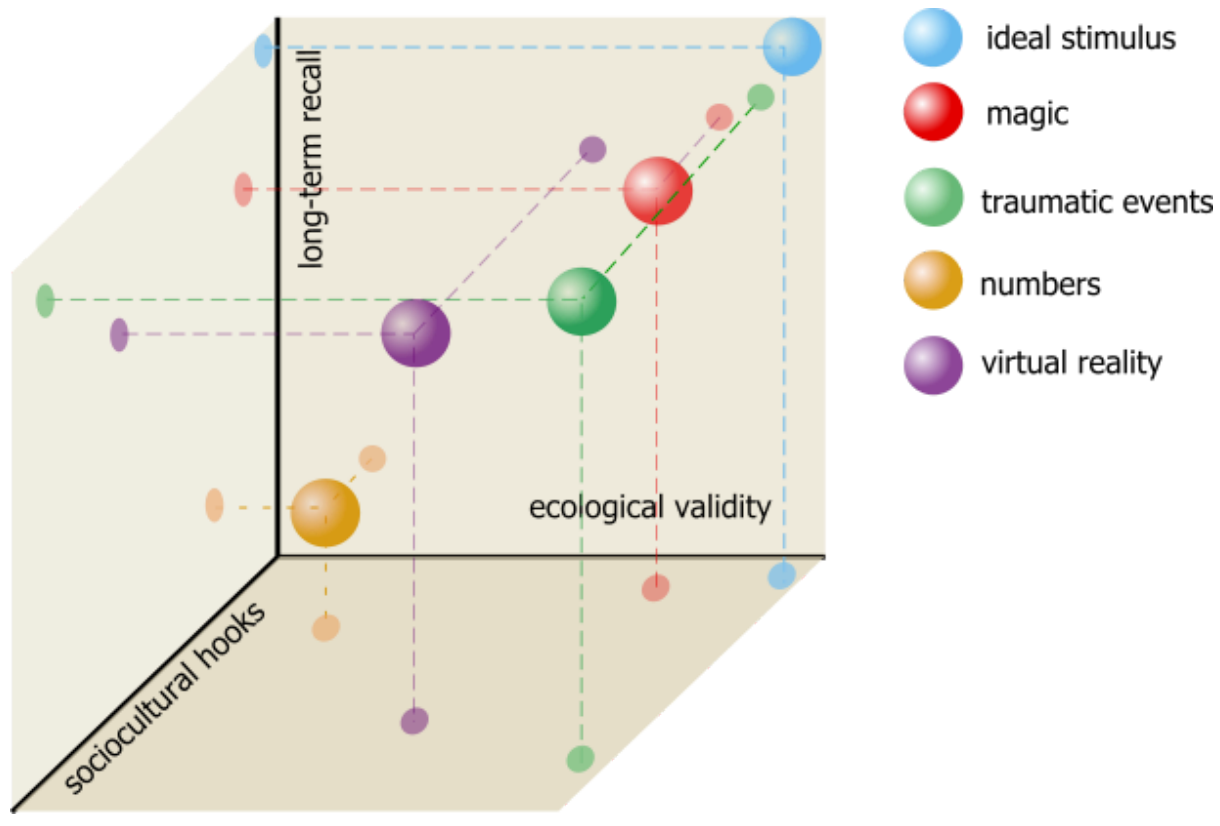

Figure 1: Magic tricks as stimuli to study episodic memory. Comparison of magic tricks with other stimuli to study episodic memory: they have the closer theoretical spatial distance towards the ideal stimulus: maximal ecological validity and long-term recall and minimal sociocultural hooks.

Magic tricks fulfill most of the requirements previously exposed to study episodic memory. In Fig. 1, we provide a schematic view of the relevant aspects we think distinguish magic from other stimuli: First, magic tricks are much more ecological (reproducing a real event) than lists of numbers or virtual reality environments. Second, magic tricks apparently have stronger long-term recall compared to numbers or ordinary events and, finally, due to the feature of impossibility of magic tricks, they are less likely to generate sociocultural 
associations that facilitate their posterior spontaneous retrieval. This last point is opposed to traumatic events which have both elevated recall and ecological validity but also strong sociocultural associations. Taking these three domains into account, we think magic tricks could have the closest theoretical distance to the ideal stimulus to study episodic memory.

Magic tricks have been used in neuroscience to explore attention (Kuhn \& Teszka, 2015); perception (Ekroll et al., 2017) or decision-making (Shalom et al., 2013; Olson et al., 2015) but they have never been used experimentally to study memory processes. Magicians are aware of the relevance of memory processes for creating their tricks. Juan Tamariz, a renowned Spanish magician pioneer in the role of memories in magic tricks wrote: "The magician has to know how to cause gaps in the memory of the spectators to make them forget what we want for the magic effect, or make them believe they remember things that did not really exist..." (Tamariz, 1988). The intuition of Tamariz that magic tricks interact with all sorts of memory processes of the audience has just been recently supported by theoretical neuroscience (Macknik et al., 2008; Quian Quiroga, 2016; Bestue, 2019; Camí et al., 2020).

Magic effects are relatively untapped tools to evaluate several cognitive processes, including those that involve episodic memory (Camí et al., 2020). Little is known about memory decay of magic tricks -which parts or details are best remembered- or the relevance of serial-position effects. These phenomena are of high interest both for neuroscience and magic. The serial-position effects were described for the first time by Hermann Ebbinghaus (Ebbinghaus, 1885/1913) and they state that the memory recall of an element varies as a function of its position inside the sequence. In the context of episodic memory, primacy and recency effects (the first ones or the last ones present higher recall) have been reported in different contexts such as TV ads (Terry, 2005; Li, 2009), chronological memories (Roediger \& Crowder, 1976), parking locations (Da Costa Pinto \& Baddeley, 1991), music (Overstreet \& Healy, 2010) or internal mentation (Stawarczyk \& D'Argembeau, 2019). However, rates of recall are really low in the long-term, so studying how serial-position effects will affect pure episodic memories in the long-term is challenging. Indeed, many magicians have elaborated their own theories about how to structure magic shows, but these magician's theories have never been tested experimentally. Studying how the position of a magic trick inside the show affects recall can be beneficial both for neuroscience and magic. Here, apart from studying memory decay of magic tricks and its saliences, we particularly checked if any serial-position effect was observed in magic tricks at different times, from just after the show up to 4.5 months.

\section{Methods}

A professional magician (one of the authors, MAG) performed a real magic show of approximately 45 minutes that consisted of eight different magic tricks (trick\#1 - trick\#8, Supplementary material). We define a trick as an illusory feat containing a single or multiple 
moments of apparent impossibility under a common plot motif. To preserve the ecological relevance of the experiment and avoid the Hawthorne effect (Mayo, 1933), we did not study each trick in isolation, as we would in a laboratory setting, but as part of a conventional magic show performed live in front of the audience. The show included both coins and card tricks which differed in complexity. They all belong to the professional repertoire of the magician, usually performed in front of lay people. The tricks were performed in front of three groups of participants ( $n=118,62$ female, $M=40.6, S D=15.7$ ). Each group witnessed all eight tricks and had no interaction with the other groups. The whole experiment was run within the same day (30th October 2019, Barcelona), in the same room, and with consecutive sessions. Participants of one group were also instructed not to interact with other participants, and were asked to leave the room through a different door to minimize any possible interaction. Two out of the three groups ( $n=76$ : onward\#1=38, onward\#2=38) viewed the tricks in the onward sequence (trick\#1 - trick\#8) while the remaining group $(n=41)$ viewed the same tricks in backward sequence (trick\#8 - trick\#1). We used two onward sessions to test memory robustness of magic tricks as well as for introducing minor modifications not addressed in this publication (Supplementary material). Unbeknownst to the participants in advance, at the end of the show they were given a blank sheet of paper where they had to individually and freely recall the tricks they remembered to have just seen, under the instruction "What tricks do you remember?". After 10 days, they received an unexpected email asking them to list again the tricks they remembered $180.3 \%$ answered, $n=94$ ). Another email was sent after 1.5 months ( 45 days, $60.7 \%$ answered, $n=71$ ) and a final one was sent 4.5 months after the show (135 days, 44.9\% answered, $n=53$ ) asking participants to list the tricks they remember. In the online interactions, participants were explicitly asked not to check the chain of previous emails. A schematic design of the experiment is illustrated in Fig. 2.
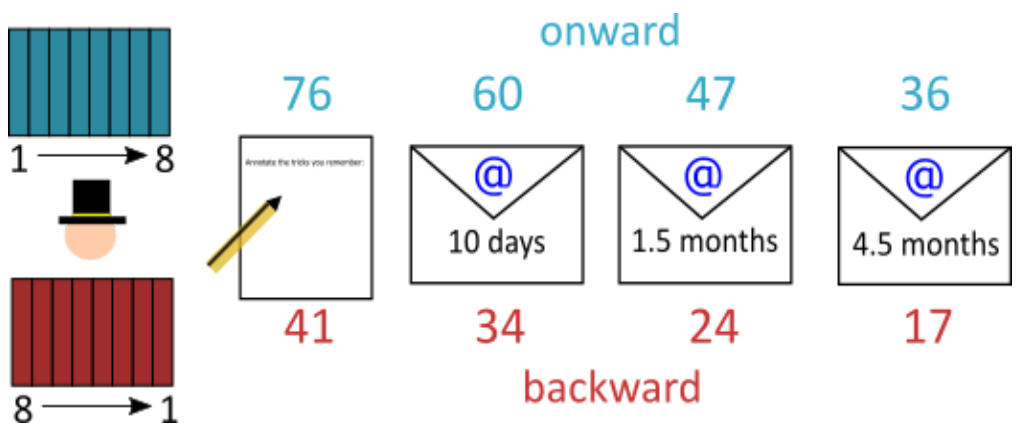

17
Figure 2. Experimental design. Total amount of responses from participants who viewed the onward sequence (in blue, onward\#1 + onward\#2) and total amount of responses for participants who viewed the backward sequence (red). Subjects were asked to report the tricks they remembered four times: just after the show, 10 days after, 1.5 months after and 4.5 months after. Except for the first one, which was done onsite, the rest of the memory tests were carried out by email.

For the analysis, each trick was considered as a binomial variable: "remembered""not-remembered". We considered that a trick was "remembered" by a spectator when at least one of its saliences was reported. A salience is defined as any concrete aspect of the 
performance (prediction, travel, disappearance, plot, props used, etc.) that makes reference unambiguously to a particular trick (see Supplementary material).

The analysis was computed with mixed logistic regressions models. We used them to evaluate recall over time and the models fitted an intercept for each subject. For group comparisons, we used the two-proportion z-test for groups of $n=2$ and the Dunn's test correcting for multiple comparisons for groups of $n>2$. Multiple comparisons tests were computed using the statsmodel package of python, version 0.9.0. For the mixed logistic regression model, we used the $\mathrm{R}$ package Ime4. For all cases, $\mathrm{a}=0.05$.

In accordance with ethical standards, procedures were approved by the institutional review board of the University Miguel Hernández from Alicante (reference 2019.264.E.OIR to AGM). All participants were over 18 y.o. that signed a consent form agreeing to be filmed during the show and also agreeing to be contacted by email again in the future.

\section{Results}

What do people remember after a magic show? Is there any serial-position effect of the tricks both in the short and the long-term? To answer these questions, eight different magic tricks were specifically selected for this experiment (trick\#1 - trick\#8) and performed by a professional magician in front of three groups of participants $(n=117)$. Two groups $(n=76)$ viewed the tricks in the onward sequence (trick\#1 - trick\#8) while the remaining group viewed the same tricks in backward sequence (trick\#8 - trick\#1). They freely recalled the tricks they remembered at the end of the show, 10 days after the show $(80.3 \%$ answered, $\mathrm{n}=94), 1.5$ months (45 days) after (60.7\% answered, $\mathrm{n}=71$ ) and 4.5 months ( 135 days) after $(40.7 \%, n=48)$. This experimental design (Fig. 2) allowed us to test memory recall for different magic tricks and look for the interaction of serial-position effects with time.

First, we evaluated the memory decay of magic tricks. We found a clear memory decay with time (mixed logistic regression: $\beta=-0.052, \mathrm{SE}=0.007, \mathrm{t}=-7.31, \mathrm{p}<0.001$ ) when collapsing the data by trick and session (Fig. 3). Differences between all times were found (multiple comparison Dunn tests; except the comparison 1.5 months - 4.5 months, $p=0.11$ ). This decay was systematic across magic tricks and sessions. Magic tricks decayed with time as standard episodic memories but still presented significant recall after 4.5 months $(t=14.33$, $p<0.001)$. 
A

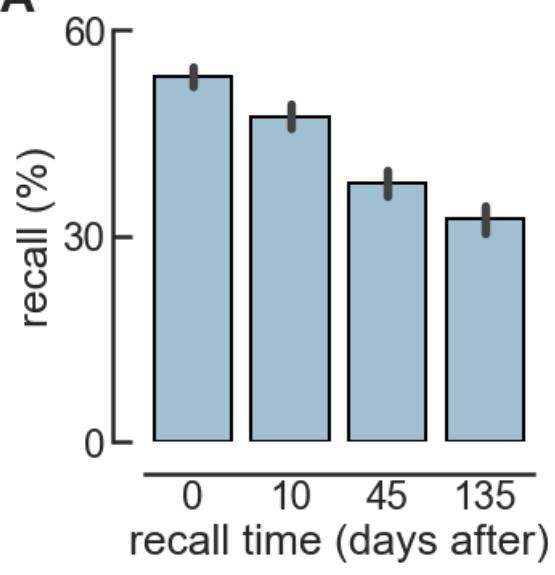

B

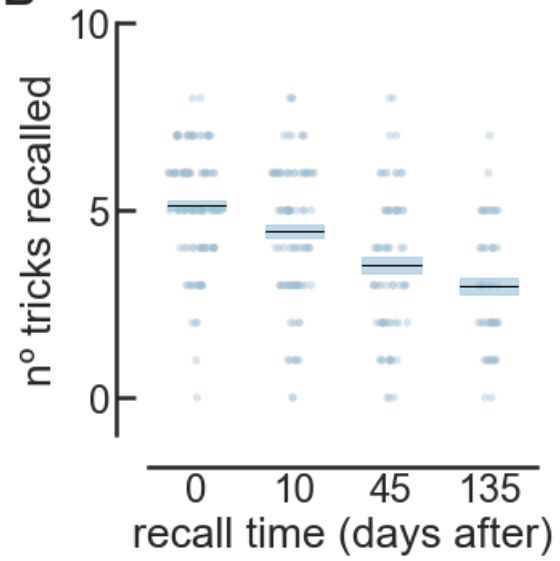

Figure 3. Memory decay. A) Percentage of tricks recalled at different times (mean and s.e.m). B) Average number of tricks remembered by each participant at different times. Each dot depicts an individual subject. The box reflects the mean and the s.e.m.. Memory decay is also observed with this measure (mixed linear regression: $\beta=-0.797, \quad S E=0.018, \quad t=-44.8$, $p<0.001)$.

Then, we checked the robustness of magic tricks to study memory-related effects. We compared, trick by trick, the two groups that viewed the tricks in the onward sequence (onward\#1 and onward\#2). No difference was observed (multiple comparison Dunn test) for any tricks between the two groups, showing strong stability of the inherent memorability of each trick. Compared to the group that viewed the same tricks in backward sequence, statistical differences were observed for tricks \#1, \#3, \#7 and \#8 (Supl. 1 and Supl. 2). Together, these results illustrate that tricks have a different value of intrinsic memorability that gives a stable average of recall. The stability observed trick by trick allowed us to merge the two groups that viewed the onward sequence and compare them with the one that viewed the backward sequence (Supl. 3) for the coming analysis of the serial-position effect.

We then investigated the role of the sequence order (onward/backward) in the memorability of each magic trick after the show. We found that sequence direction enhanced the recall of the last trick performed (trick\#8 in onward sequence and trick\#1 in backward sequence). This recency effect (Fig. 4, left) was quantified with a two-proportion z-test (trick\#1: $z=4.15, p<0.001$ \& trick\#8: $z=-5.53, p<0.001$ ). The only trick that presented differences was trick\#7, in the same direction as trick\#8 $(z=-2.97 \& p=0.003)$. These results show that, for most of the tricks, the memorability of the trick is independent of the serial-position and just depends on intrinsic memorability (strength of the effect, hookable saliences, etc..). However, those tricks placed at the end of the show benefit from recency effects only right after the show, independently of its intrinsic memorability. 

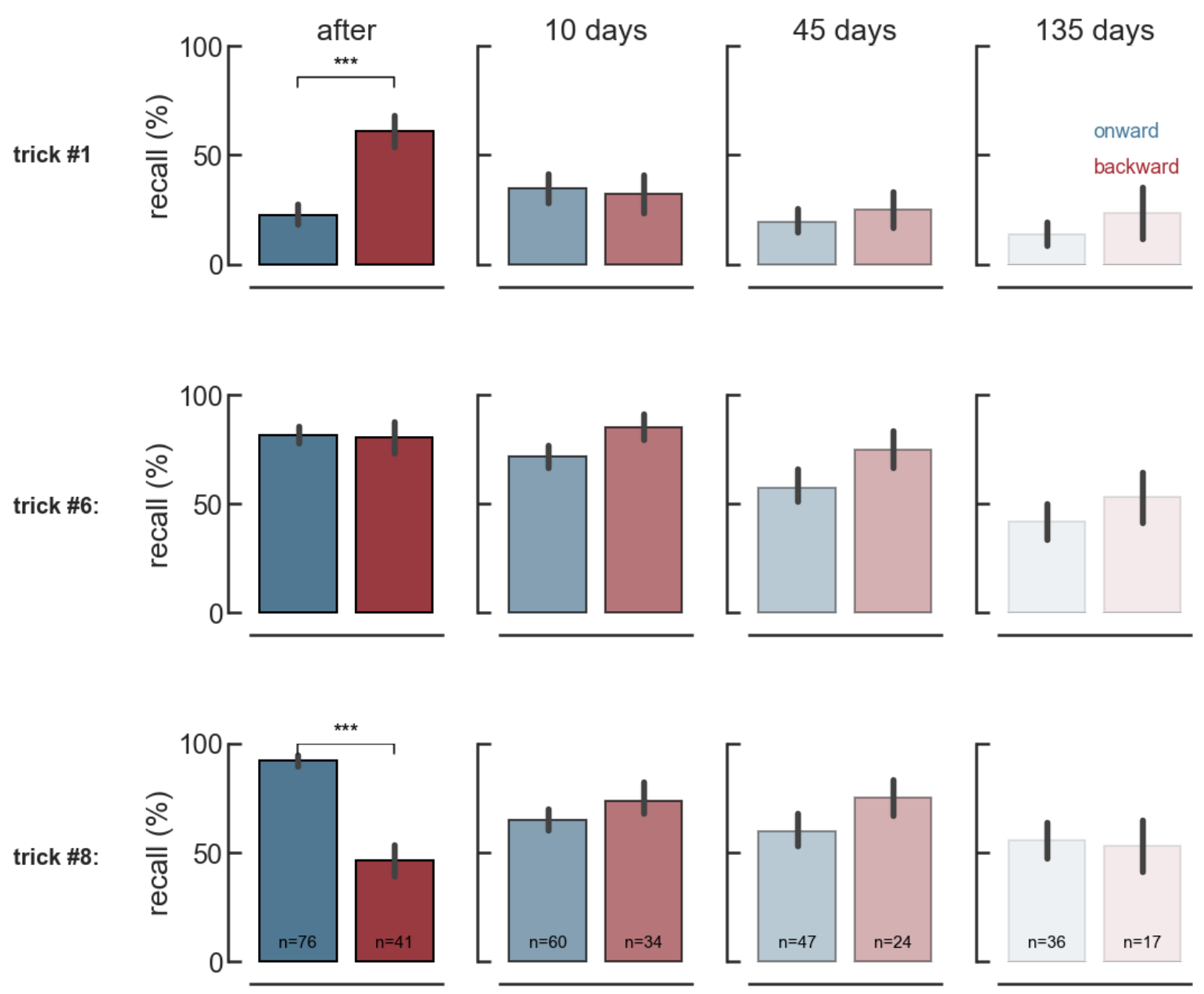

Figure 4. Recency effects are not maintained in the long-term. Recall for the first and the last trick (trick\#1 and trick\#8) of the session with time splitted by sequence order (onward/backward). Memory gain observed just after the show (left) for the last trick in each sequence order. This effect vanishes with time (right), resulting in significant interactions order-time when tested with a mixed logistic regression (Supl. 4 and Supl. 5). Trick\#6 is also included as an example of an intermediate trick. Tricks in the middle that are equally maintained after 4.5 months do not present the interaction order-time (trick\#6: $\beta=0.35, \mathrm{SE}=0.38, \mathrm{z}=0.94, \mathrm{p}=0.35)$.

The previously reported short-term memory gain (recency) made us wonder how this effect evolved with time. To evaluate that, we checked the interaction in each trick between sequence order (onward/backward) and time (just after, 10 days and 1.5 months and 4.5 months). No interaction would imply standard monotonic decay, while an interaction would indicate that short-term memory gains do not necessarily translate into long-term memory gains for episodic memories. When modeling the recall (binomial) as a function of the interaction sequence order (onward/backward) - time (after/10/45/135 days), the interaction was significant for trick\#1 and trick\#8 ( $\beta=-1.07, \mathrm{SE}=0.4, z=-2.66, p=0.008$ and $\beta=1.27, \mathrm{SE}=0.33, z=3.8, p<0.001$, respectively), revealing a recency effect of position that does not translate into long-term memory gains (Fig. 4). The detailed model can be found in 
Supl. 4 and Supl. 5. This analysis was dissected by saliences (Supl. 6 and Supl. 7), revealing that order effect holds even when removing the "trick" construct. Both trick\#1 and trick\#8 were significantly remembered after 4.5 months (trick\#1 onward: $t=2.3, p=0.02$, backward: $t=2.13, p=0.03$; trick\#8 onward: $t=5.26, p<0.001$, backward: $t=3.5, p<0.001$ ). This was not the case in trick\#2, which was not significantly remembered in either session after 4.5 months (trick\#2 onward: $t=1.77, p=0.07$, backward: $t=1.46, p=0.145$ ) nor in trick\#4 (Supl. 8), whose recall for the backward session was not significantly different from 0 (trick\#4 backward: $t=1.02, p=0.31$ ). In trick\#4, we also found a significant interaction order-time $(\beta=-1.07, \mathrm{SE}=0.46, \mathrm{t}=-2.3, \mathrm{p}=0.02)$. In this trick, no differences are observed after the show $(t=0.58, p=0.56)$, so the interaction is driven by memory extinction instead of serial-position effects (as in trick\#1 and trick\#8). Besides trick\#1, trick\#4 and trick\#8, no other trick presented the interaction order-time. In sum, our results clearly show that long-term episodic memory was not affected by serial-position effects. Instead, their recall depended solely on the intrinsic memorability of each trick.

\section{Discussion}

Magic tricks are an invaluable tool to study cognitive processes in the real world (Camí et al., 2020), such as episodic memories as we did with the current study. In Pause et al. (2013), seven criteria are proposed to investigate episodic memory. Surprisingly, magic tricks in the context of a real show accomplish all seven: first, they can be tested and manipulated under laboratory conditions. Second, they are not explicitly instructed to be memorized. Third, they have an intrinsic emotional component. Fourth, they are a one-trial learning event. Fifth, they include: what, where and when information. Sixth, the context of the show makes the memory test unexpected. Finally, the test can be performed after a long retention interval with elevated recall. Regarding the last point, we observed high rates of recall after 4.5 months. Other studies have found significant recall in the long-term (da Costa Pinto \& Baddeley, 1991; Hirst et al., 2009) but magic tricks have less sociocultural hooks and are harder to remember (card tricks in particular, as recently suggested by the magician Joshua Jay (Jay, 2016). Magic tricks are dissonant situations with no influence on our lives, so are less likely to be retrieved on a daily basis (Steinkraus, 1979; Camí \& Martínez, 2020). Regarding the strength of magic tricks for future memory experiments, they are complex ecological stimuli, they have minimal sociocultural associations, minimizing the probability of sporadic retrievals, and they present elevated maintenance in the long-term.

When addressing serial-position effects, we found recency effects just after the show that were not maintained in the long-term. With this result, we described how serial-position effects evolve with time when using real-world episodes. Previous studies of working memory reported that introducing a task that demanded cognitive effort before the recall cancelled recency effects (Postman \& Phillips, 1965; Glanzer \& Cunitz, 1966; Bjork \& Whitten, 1974). However, when this effect is present in recall, the gains should also be 
reflected in the long-term if we consider a monotonic decay of memory (Roediger \& Crowder, 1976; da Costa Pinto \& Baddeley, 1991; Grillon et al., 2008; Souza \& Oberauer, 2017). Compared to previous studies, ours showed elevated memory recall (Misra et al., 2018) with minimal sociocultural hooks (da Costa Pinto \& Baddeley, 1991). Bjork and Whitten (1974) glimpsed the disappearance of serial-position effects after 24 hours, but it was done with sequences of numbers and minimal recall. When looking at more stable sequential memories in the long-term, as the list of presidents of the USA (Roediger \& Crowder, 1976), primacy and recency effects are reported, evidencing that naturalistic episodic events are stored in a different way than learned sequences (Davachi \& DuBrow, 2015). The main implication of the study regards the relation between short-term and long-term memory: previous studies observed that increased short-term memory maintenance lead to stronger long-term memory recall (Hartshorne \& Makovski, 2019, meta-analysis) as well as predicted a monotonic decay of long-term memory with time (Ebbinghaus, 1885/1913; Wixted \& Carpenter, 2007). Our results, however, point towards a model where short-term mechanisms provide a gain to the intrinsic memorability of the event and where long-term memory decay is independent of these gains and has its starting point in the intrinsic memorability of the event. Taken together, our results provide new insights into the standard short-term memory and long-term memory interplay (Atkinson \& Shiffrin, 1968; Ericsson, 1995; Baddeley, 2000) to include this intrinsic memorability of the events in episodic memory. However, larger sample sizes and more detailed delays must be included before exploring new models of memory decay.

A main limitation of our study is that participants were interrogated four times (just after/10/45/135 days), and not just one as proposed in Pause et al. (2013). Acknowledging the possibility of a slight anchoring effect (participants were subsequently reasked by email), the fact that episodic memory decays lessens this concern while illustrating the suitability of magic shows to study memory. A secondary analysis excluding the recalls 1.5 and 4.5 months later still found the significant interaction order-time just for trick\#1 and trick\#8 $(\beta=-3.87, \mathrm{SE}=1.22, \mathrm{z}=-3.17 \mathrm{p}=0.002$ and $\beta=4.11, \mathrm{SE}=1.07, \mathrm{z}=3.83, \mathrm{p}=0.001$, respectively), indicating that the observed short-term memory gains from serial-position effects disappeared soon after the show further suggesting no anchoring effects. Other limitations came due to the nature of magic tricks: magic tricks can not be done twice, as knowing the final result ruins the surprise component of the trick. For that reason, we could not compare the onward session to the backward session in the same subjects, which would help to reduce subject variability. However, the fact that no differences in memorability were found between sessions one and three in any trick (both onwards, Supl. 2) makes us think subject variability is small when using magic tricks as stimuli. A final limitation regards the complexity of magic tricks: magic tricks trigger simultaneously many cognitive processes (Bestue, 2019; Camí et al., 2020) so modeling approaches should be taken carefully due to possible interactions with other cognitive processes. 
These results also have implications for the magic community. Magic theories are formulated without previous hypotheses (a posteriori) and from subjective data (the magician evaluates if the spectator has enjoyed the trick). This trial-and-error methodology generates many untested theories regarding the same topic, and the order of the effects in a show is not an exception. For attentional and theatrical purposes, the first and last trick are always supposed to be stronger than the rest (Giobbi, 1996; Ortiz, 1995; Tamariz, 2019). However, this hypothesis is not extrapolable to memory, as recall decayed to baseline independently of the order. Corroborating the idea that attentional/theatrical techniques regarding the order do not necessarily increase memorability, no primacy effect was observed here. An important point of the study is the style of the magician, who performed with no theatrical link between the tricks (like singers with their songs). This fact was essential to study serial-position effects, as we wanted to avoid fomenting temporal clustering (Kahana, 1996). However, we hypothesize that under a dramatic structure that links the tricks, different results might be found. In this line, a proposed future experiment would be to theatrically link the tricks and to check if this modification changes the results.

In sum, memory decay was found to be independent of short-term related memory gains. The disappearance of this recall gain with time clearly shows that the starting point of memory decay does not originate from the combination of short-term and long-term mechanisms. Future memory models have to be developed to account for this intriguing phenomena. The fact that magic tricks memorability had never been studied in a longitudinal way (repeated observations of the same subjects) up to this point, opens up the door to inspect other theories, such as the role of emotion in memory (Phelps, 2006) or the formation of false memories (Loftus \& Pickrell, 1995). Future research may find in magic not just a valuable tool for episodic memory rather an unexplored avenue to study cognition in the real world.

\section{Acknowledgements}

This work was funded by the Spanish Ministry of Science (RYC-2017-23599 grant to Alex Gomez-Marin).

\section{Data and code availability}

The tricks were performed by magician Miguel Angel Gea (co-author and the professional magician who carried out the show). The selection of the tricks was consensuated between the researchers and the professional magician. A video of the complete show (corresponding to the onward\#1 group) can be found here:

\section{https://youtu.be/b1YZutaGmvU}

Raw data (extracted saliences across time and participant) and codes (both for the analysis of the data and the generation of the figures) can be found in the following Github repository:

\section{https://github.com/davidbestue/episodic-memory-magic}




\section{References}

Alberini, C. M. (2011). The role of reconsolidation and the dynamic process of long-term memory formation and storage. Front. Behav. Neurosci. 5, https://doi.org/10.3389/fnbeh.2011.00012

Atkinson, R. C. \& Shiffrin, R. M. (1968). Human Memory: A Proposed System and its Control Processes. Psychology of Learning and Motivation (Vol. 2, pp. 89-195). Elsevier.

Bestue, D. (2019). Model of Information Process for Neuromagic. PsyArXiv, July 31. doi:10.31234/osf.io/d374z.

Bjork, R. A. \& Whitten, W. B. (1974). Recency-sensitive retrieval processes in long-term free recall. Cognitive Psychology, 6(2), 173-189.

Camí, J., Gomez-Marin, A. \& Martínez, L. M. (2020). On the cognitive basis of illusionism. PeerJ 8:e9712. DOI 10.7717/peerj.9712

Camí, J. \& Martínez L.M. (2020) El Cerebro Ilusionista. RBA, Barcelona.

Chow, T. E. \& Rissman, J. (2017). Neurocognitive mechanisms of real-world autobiographical memory retrieval: $\quad$ insights from studies using wearable camera technology. Ann NY Acad Sci 1396, 202-221, https://doi.org/10.1111/nyas.13353

Da Costa Pinto, A. \& Baddeley, A. D. (1991). Where did you park your car? Analysis of a naturalistic long-term recency effect. European Journal of Cognitive Psychology, 3(3), 297-313.

Davachi, L. \& DuBrow, S. (2015). How the hippocampus preserves order: the role of prediction and context. Trends in Cognitive Sciences, 19(2), 92-99.

Ebbinghaus, H. (1913). Memory: a contribution to experimental psychology (transl. H.A. Ruger \& C.E. Bussenvis). New York, NY: Teachers College (Original work published in 1885).

Ekroll, V., Sayim, B., Wagemans, J. (2017). The Other Side of Magic: The Psychology of Perceiving Hidden Things. Perspect Psychol Sci. 2017;12:91-106.

Giobbi, R. (1996). Card College - Volume 2. Seattle, Hermetic Press.

Glanzer, M. \& Cunitz, A. R. (1966). Two storage mechanisms in free recall. Journal of verbal learning and verbal behavior, 5(4), 351-360.

Gold, P. E., McIntyre, C., McNay, E., Stefani, M., Korol, D. L. (2001). Neurochemical referees of dueling memory systems. Memory consolidation: Essays in honor of James L. McGaugh. pp.219. doi:10.1037/10413-012. ISBN 978-1-55798-783-9.

Grillon, M. L., Johnson, M. K., Krebs, M.-O., \& Huron, C. (2008). Comparing effects of perceptual and reflective repetition on subjective experience during later recognition memory. Consciousness and Cognition, 17(3), 753-764.

Hackman, Daniel A.; Betancourt, Laura M.; Gallop, Robert; Romer, Daniel; Brodsky, Nancy L.; Hurt, Hallam; Farah, Martha J. (2014). "Mapping the Trajectory of Socioeconomic Disparity in Working Memory: Parental and Neighborhood Factors". Child Development. 85 (4): 1433-1445

Hardt, O., Einarsson, E. O., Nader, K. (2009). A bridge over troubled water: reconsolidation as a link between cognitive and neuroscientific memory research traditions. Annu. Rev. Psychol. 61,141-167. 
Hargreaves, I. S., Pexman, P. M., Johnson, J. C., \& Zdrazilova, L. (2012). Richer concepts are better remembered: Number of features effects in free recall. Frontiers in Human Neuroscience, 6, Article 73.

Hebb, D. O. (1961). Distinctive features of learning in the higher animal. In J. F. Delafresnaye (Ed.), Brain mechanisms and learning: A symposium (pp. 37-51). Oxford: Blackwell Scientific.

Hirst, W., Phelps, E. A., Buckner, R. L., Budson, A. E., Cuc, A., Gabrieli, J. D. E., Johnson, M. K. (2009). Long-term memory for the terrorist attack of September 11: flashbulb memories, event memories, and the factors that influence their retention. Journal of Experimental Psychology. General, 138(2), 161-176.

Horner A. J., Bisby J. A., Bush D., Lin W. J., Burgess N. (2015). Evidence for holistic episodic recollection via hippocampal pattern completion. Nature Communications.6:7462.

Howieson, D. B., Mattek, N., Seeyle, A. M., Dodge, H. H., Wasserman, D., Zitzelberger, T., \& Jeffrey, K. (2011). Serial position effects in mild cognitive impairment. Journal of clinical and experimental neuropsychology, 33(3), 292-299.

Jay, J. (2016). What do audiences really think?, Magic, 25(13), pp. 46-55.

Kahana, M.J. (1996). Associative retrieval processes in free recall. Mem.Cognit. 24, 103-109.

Kuhn, G. \& Teszka, R. (2015). Attention and misdirection: How to use conjuring experience to study attentional processes. In: Fawcett JM, Risko EF \& Kingstone A, editors. The Handbook of Attention. Cambridge, Massachusetts: MIT Press; 2015. p 503-25

Li, C. (2009). Primacy effect or recency effect? A long-term memory test of Super Bowl commercials. Journal of Consumer Behaviour.

Loftus, E. F., \& Pickrell, J. E. (1995). The formation of false memories. Psychiatric Annals, 25(12), 720-725.

Macknik, S., King, M., Randi, J., Robbins, A., Teller, Thompson, J., Martinez-Conde, S. (2008). Attention and awareness in stage magic: turning tricks into research. Nat Rev Neurosci. 2008;9:871-9.

Mayo, E. (1933). The Human Problems of an Industrial Civilization. Pp.194. New York: The Macmillan Company.

Misra, P., Marconi, A., Peterson, M., \& Kreiman, G. (2018). Minimal memory for details in real life events. Scientific Reports, 8(1), 16701.

McGaugh, J. L. \& Roozendaal, B. (2002). Role of adrenal stress hormones in forming lasting memories in the brain. Current Opinion in Neurobiology. 12 (2): 205-210. doi:10.1016/S0959-4388(02)00306-9. PMID 12015238

Olson, J. A., Amlani, A. A., Raz, A., Rensink, R. A. (2015). Influencing choice without awareness. Consciousness and Cognition, 37, 225-236.

Overstreet, M.F., Healy, A.F. (2011). Item and order information in semantic memory: students' retention of the "CU fight song" lyrics. Mem Cogn 39, 251-259

Ortiz, D. (1995). Strong Magic. Kaufman and Company, USA.

Pause, B. M., Zlomuzica, A., Kinugawa, K., Mariani, J., Pietrowsky, R., Dere, E. (2013). Perspectives on episodiclike and episodic memory. Frontiers in Behavioral Neuroscience, 7, 33.

Phelps, E. A. (2006). Emotion and cognition: insights from studies of the human amygdala. Annu. Rev. Psychol., 57, 27-53. 
Plancher, G. \& Piolino, P. (2017). Virtual reality for assessment of episodic memory in normal and pathological aging, in The Role of Technology in Clinical Neuropsychology, eds T. Parsons and R. Kane (Oxford: $\quad$ Oxford University Press).

Postman, L. \& Phillips, L. W. (1965). Short-term Temporal Changes in Free Recall. Quarterly Journal of Experimental Psychology, 17(2), 132-138.

Quian Quiroga, R. (2016). Magic and cognitive neuroscience. Current Biology, 26(10), R390-4.

Roediger, H. L. \& Crowder, R. G. (1976). A serial position effect in recall of United States presidents. Bulletin of the Psychonomic Society, 8(4), 275-278.

Sara, S. J. (2000). Strengthening the shaky trace through retrieval. Nat. Rev. Neurosci. 1, 212-213.

Steinkraus, W. E. (1979). The art of conjuring. Journal of aesthetic education, 13, 17-27.

Tamariz, J. (1988). Fundamentos del ilusionismo, en Secretos de Magia Potagia, Editorial Frakson, Madrid.

Tamariz, J. (2019). The Magic Rainbow, Seattle, Hermetic Press.

Tulving, E. (1972) Episodic and semantic memory. In Organisation of Memory (Tulving, E. and Donaldson, W., eds), pp. 381-403, Academic Press.

Shalom, D.E., de Sousa Serro, M.G., Giaconia, M., Martínez, L.M., Rieznik, A., Sigman, M. (2013). Choosing in Freedom or Forced to Choose? Introspective blindness to psychological forcing in stage-magic. PLoS One. 2013;8(3)e58254.

Souza, A. S., \& Oberauer, K. (2017). Time to process information in working memory improves episodic memory. Journal of Memory and Language, 96, 155-167.

Terry, W. S. (2005). Serial position effects in recall of television commercials. The Journal of general psychology, 132(2), 151-163.

Wixted, J. T., \& Carpenter, S. K. (2007). The Wickelgren Power Law and the Ebbinghaus Savings Function. Psychological Science, 18(2), 133-134. 


\section{Supplementary material}

\section{Supplementary text: Participants and Experiment}

The experiment was advertised via posters in the campus of the Pompeu Fabra University of Barcelona as a "cognitive experiment in magic" (no relation to memory was ever made). The whole experiment was run within the same day (30th October 2019, Barcelona), in the same room, and with consecutive sessions (16:00, 17:30 and 19:00). Regarding the participants that will participate in the experiment, we assumed they enjoy attending magic shows, as no monetary retribution was given and that they will collaborate with us, as an experimental component was clearly announced.

A total of 117 eligible people contacted us via email in order to participate. All participants had to be adults (over 18) and needed to know Spanish (the magic show was in Spanish). Out of the 117 participants, there were 8 expert magicians who knew most of the secrets (excluding them from the analysis did not affect the results). Participants assigned themselves to one of the three sessions, so they were self distributed according to their preferred time slot (no experimental criteria for the division). We never mentioned any differences between the sessions, so participants had no incentive to assign themselves to one session or to another. Participants of one session were also instructed not to interact with other participants, and left the room through a different door to minimize any possible interaction. The participants of the first session (group 1, 16:00, $n=38$ ) viewed the tricks in the onward sequence (trick\#1 - trick\#8, onward\#1). The participants of the second session (group2, 17:30, $\mathrm{n}=41$ ) viewed the same tricks in backward sequence (trick\#8 - trick\#1). The participants of the last session (group 3, 19:00, $n=38$ ) again view the tricks in the onward sequence (trick\#1 - trick\#8, onward\#2).

As the majority of participants came from the university campus (similar sociocultural level) we did not directly correct for sociocultural level. An indirect control is that no differences were observed between groups 1 and 3 (both viewed the onward session), evidencing a weak effect of subject variability. Moreover, although it has been shown that socioeconomic status has an overall impact on working memory capacities (Hackman et al., 2014), it does not seem to be related with serial-position effects (Howieson et al., 2011), which was the main topic of the study. Immediately before the performance, participants signed a consent form allowing us to film them during the show and also authorising future contact by email. The first time we asked 
about their memory regarding the show was just at the end of it. We did not anticipate they would be contacted in the future three more times asking them for what they remember about the show. To minimize biases, we never revealed the experiment involved both memory and serial-position effects.

The only difference regarding the experiment is the order they witnessed the magic tricks. In the supplementary figure \#2, we pointed out the differences between groups in each of the magic tricks. Indeed, as groups 1 and 3 witnessed the same order session and no differences were found between them, we collapsed them for the rest of the analysis. No statistical difference was observed in the mean age (group1: $M=40.5, S D=15.7$, group2: $M=39.4, S D=16.0$, group3: $M=41.9, S D=15.9$ ) between the groups (ANOVA, pval=0.70, and no significant comparison in Tukey). Finally, no statistical differences in male-female distribution (group1: female ratio=21/38, group2: female ratio=21/41, group3: female ratio=20/38) was observed either between the groups (proportion z-test pvals>0.719).

\section{Supplementary text: Magic tricks and remembered saliences}

Spectators tend to report the recall of specific aspects of the magical effects, such as disappearances or transpositions, as well as the way they were presented or the props used. We refer to these concrete aspects of the performance as "saliences". In the remainder, we will briefly describe each trick highlighting its silences (in bold):

Trick\#1: the trick starts with the magician placing an unknown blue-back card inside the box. Then, he makes piles with the deck (red-backed) and the spectator decides which one to keep and which one to discard. During this process, the magician makes magic tricks with every pile, like a magical transposition between a joker and one indifferent card or changing the color of the back of the cards. The process ends with a single card that matches the one in the box. As encore, the magician places the blue-back card in the table and the spectator picks a different red-back. The card magically changes to match the new selection.

Trick\#2: In this trick, the spectator selects a card and returns it to the deck. The magician asks the spectator how many opportunities (chances) is he granted to find the card. He does not find it in the first one, but he transforms it to the selection. He pretends to explain it to the spectator: everything is about rubbing. When you rub the card, it changes. The selected card reappears behind the ear of the spectator. By taking the selected card and the transformed one and rubbing them together, a coin appears in between (money). The magician makes the card disappear and reappear again behind the spectator's ear. Finally, a big coin appears between the four cards. The salience of the big coin is not included in the graph because it was used to explore the extinction of a memory. The results of the big coin will be used for another publication. Briefly, the only difference between the onward \#1 and 
onward \#3 consisted in including a gag after the appearance of the big coin (a technician appeared from behind the table).

Trick \#3: The magician covered four coins with four cards. They magically travelled from under the cards to finally gather together under one of them.

Trick \#4: The magician presents a deck of cards and takes out its "soul". He gives the soul of the deck to a spectator. This one reverses one card in this imaginary deck and gives it back to the magician. He merges the soul of the deck with the real deck and reveals that there is a turned card that matches the one imaginary revolved by the spectator (prediction).

Trick \#5: The magician presents four copper coins and one glass. First, the coins changed material from copper to silver. Next, the four coins travelled between hands one by one to end up in the glass. Then, they go through the table into the glass. Finally, the coins leave the glass one by one to end back in the magician's hand while they change material back to copper.

Trick \#6: Three spectators take a random card from the deck. By spelling the cards, they appear. Any card can be spelled, and it will appear, even a card from the spanish deck. In the onward\#2, the magician made a small mistake, so we decided to include it as a salience (explain).

Trick \#7: The magician presents a trick that was given to him by a good friend from the spanish city of Granada. While reciting a poem about time, he finds the four kings of the deck. Then, the rest of the deck turns blank. The magician places three white cards on top of each king in four independent packets. As the poem progresses, three of the kings vanish from their packets and assemble with the remaining one in the final packet.

Trick \#8: The magician presents a deck with handwritten instructions in each card. A card is then selected and lost in the deck. By following the instructions, the magician finds the card. The final instruction involves the spectator shout "rise", and the selected card rises from the deck.

At the end of the show, participants were given a blank paper where they had to individually freely recall the tricks they remembered under the instruction "What tricks do you remember?". Email responses to the same question were subsequently obtained 10, 45 and 135 days after the show. The audience's memory reports refer to specific details of each trick, what we called saliences, and not the tricks as a whole. Assigning these saliences to each trick is sometimes difficult and requires intimate knowledge of the magical effects and techniques involved. To minimize any potential bias, two of the authors (DB and JC), with extensive experience in magic, classified all the references in the raw responses in one of the 8 tricks and shared the classifications with the other authors. If a reference was too 
vague to be classified, it was included in one of the following categories: "unknown-cards" / "unknown-coins" (5.76\% of all the responses). The other authors examined this preliminary sorting and proposed their corrections. The remaining disagreements were set by the professional magician who approved the final decision process.

The distribution of the data was binomial for each trick: 1 (remembered) and 0 (not-remembered). The analysis of this variable was computed with mixed logistic regressions models. We used them to evaluate memory decay over time and the models fitted an intercept for each subject. For group comparisons, we used the two-proportion z-test for groups of $n=2$ and the Dunn's test correcting for multiple comparisons for groups of $n>2$. Multiple comparisons tests were computed using the statsmodel package of python, version 0.9.0. For the mixed logistic regression model, we used the $\mathrm{R}$ package Ime4. In all cases, $\mathrm{a}=0.05$.

After careful inspection, one of the authors (DB) predefined a list of words that were found specific for each salience. We then used an algorithm to find those key words in the memory reports and assign each salience to only one of the eight magic tricks. The memorability of each salience was conducted separately in the onward and backward conditions, using z-tests for onward vs backward comparisons. 


\section{Supplementary figures and tables}
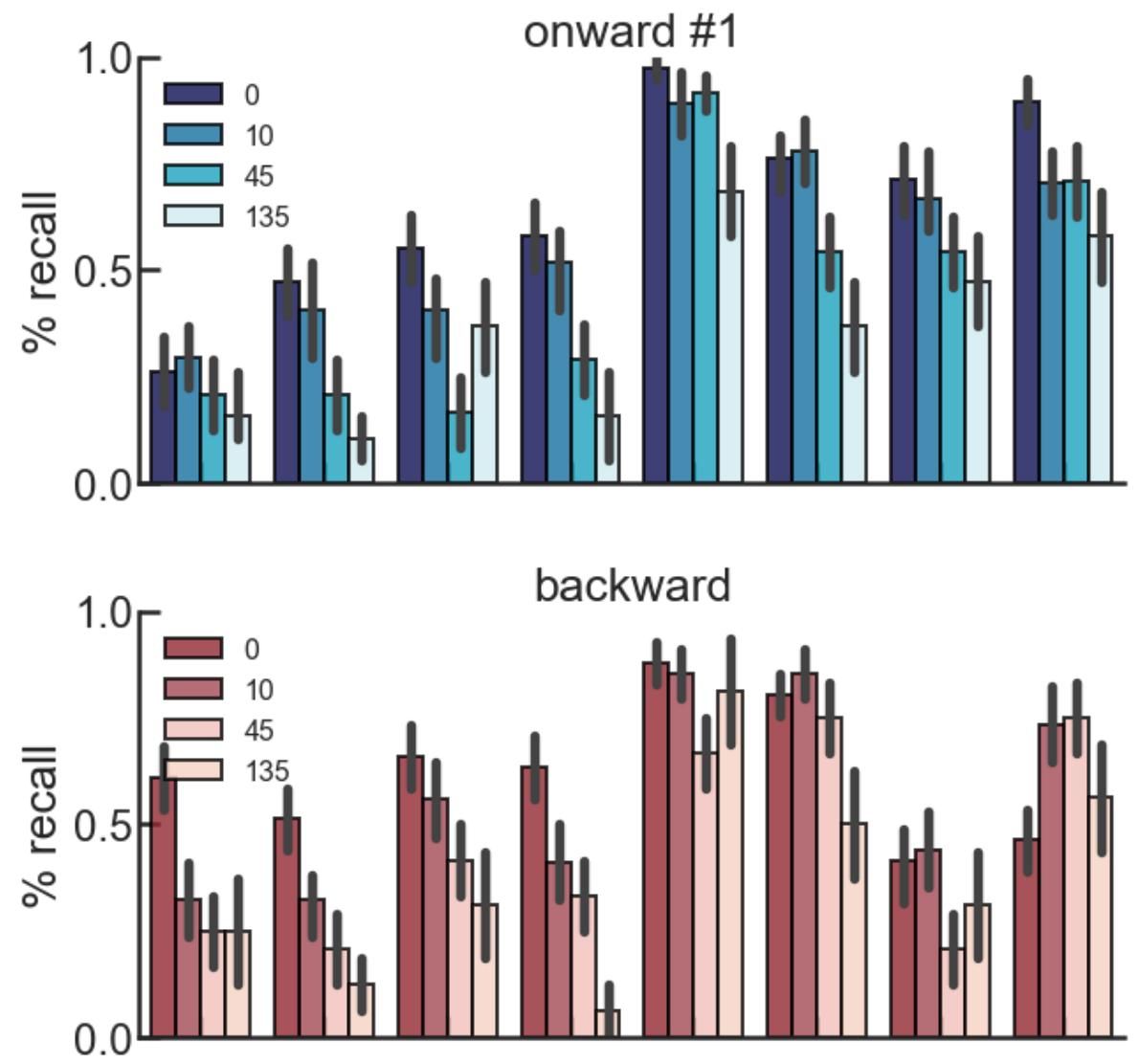

Supl. 1. Percentage of recall per trick in each session across time. All three times are reported and a systematic decay with time is systematically observed for most of the tricks. We can observe inner memorability for each trick that is stable across sessions. The recency effect is clear by comparing the trick\#8 in the onward conditions with the trick\#8 in the backward condition. The same effect occurs in trick\#1 when comparing backward with the onward sessions (error bars depict s.e.m.). High rates of recall are observed after 135 days. The intermediate tricks were essential to give the impression of a complete and realistic magic show. We want to highlight that each trick has its inner memorability: depending on the strength of

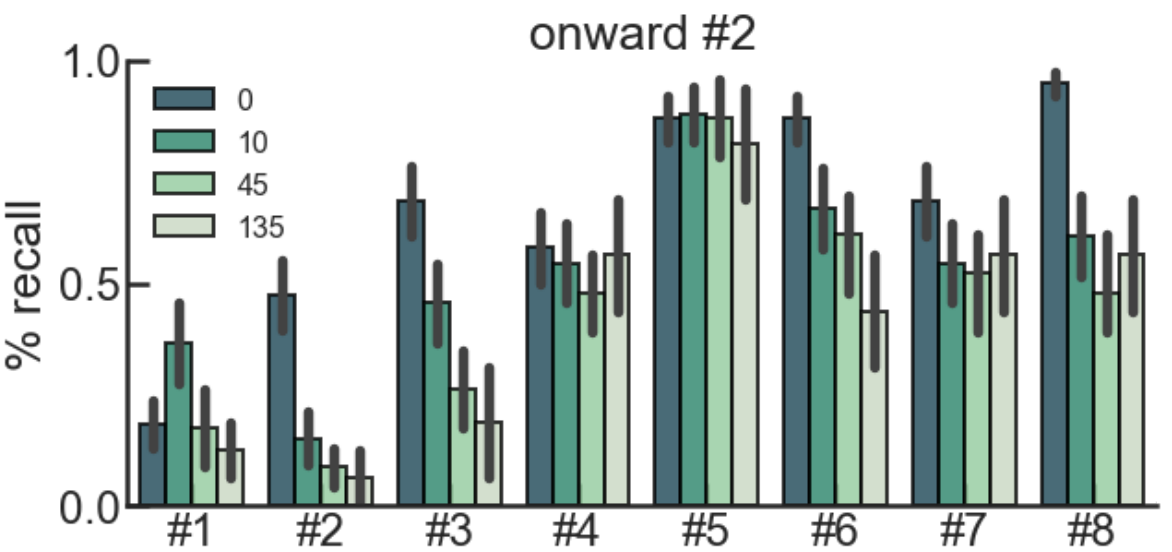
the effect, memorability is higher or lower, suggesting that intermediate effects still conserve their independence. Besides, they show the typical memory decay with time consistently across sessions, contributing to the robustness of magic tricks as valid methodological elements to study episodic memory. 


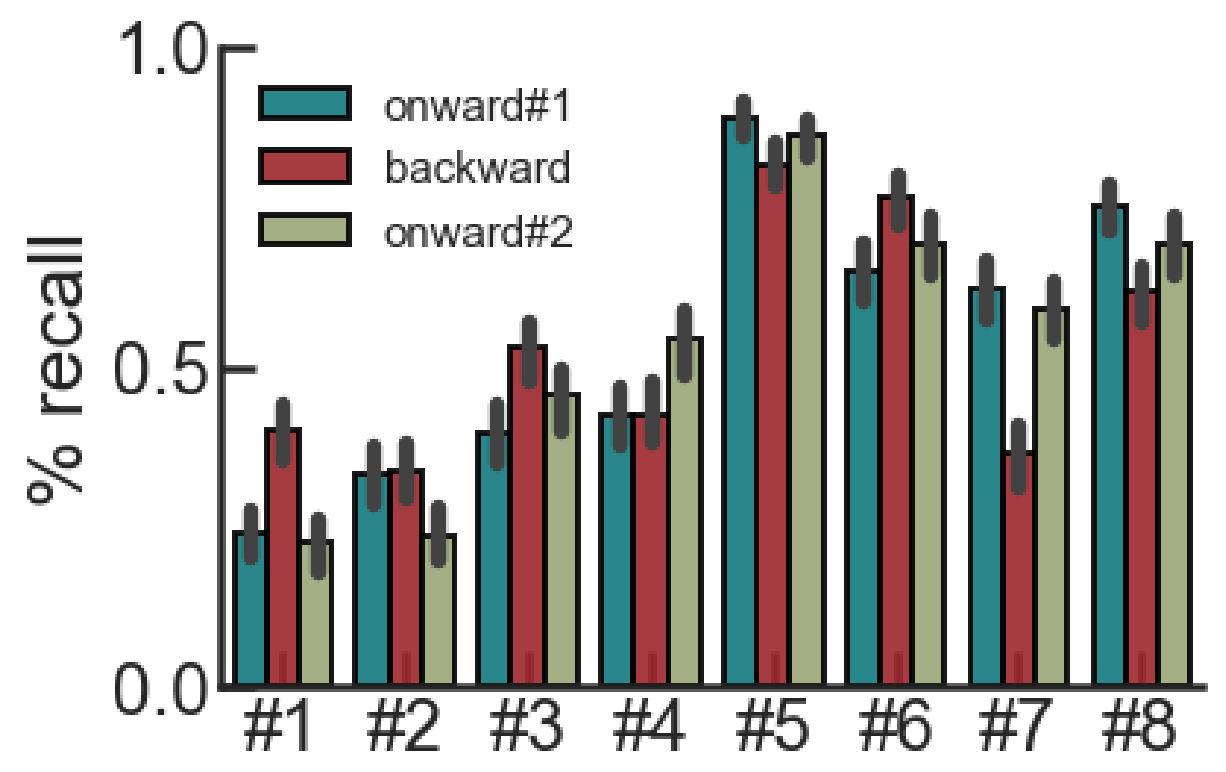

Supl. 2. Session comparison. Memory stability in onward session for all tricks. Differences were just found with backward session. Results collapsed by time. Multiple comparison Dunn test (p1-2: onward\#1- backward, p1-3: onward\#1-onward\#2, p2-3: backward-onward\#2) reveals significant difference for trick\#1 (p1-3=0.687 p1-2=0.016, p2-3=0.005), for trick\#3 (p1-3=0.438, p1-2=0.041, p2-3=0.205), for trick\#7 (p1-3=0.607, p1-2<0.001, p2-3=0.001) and for trick\#8 (p1-3=0.301, $\mathrm{p} 1-2=0.027, \mathrm{p} 2-3=0.242$ ). Critically, no difference was found between onward\#1 and onward\#2 in any trick. All differences found were with backward sequence. 

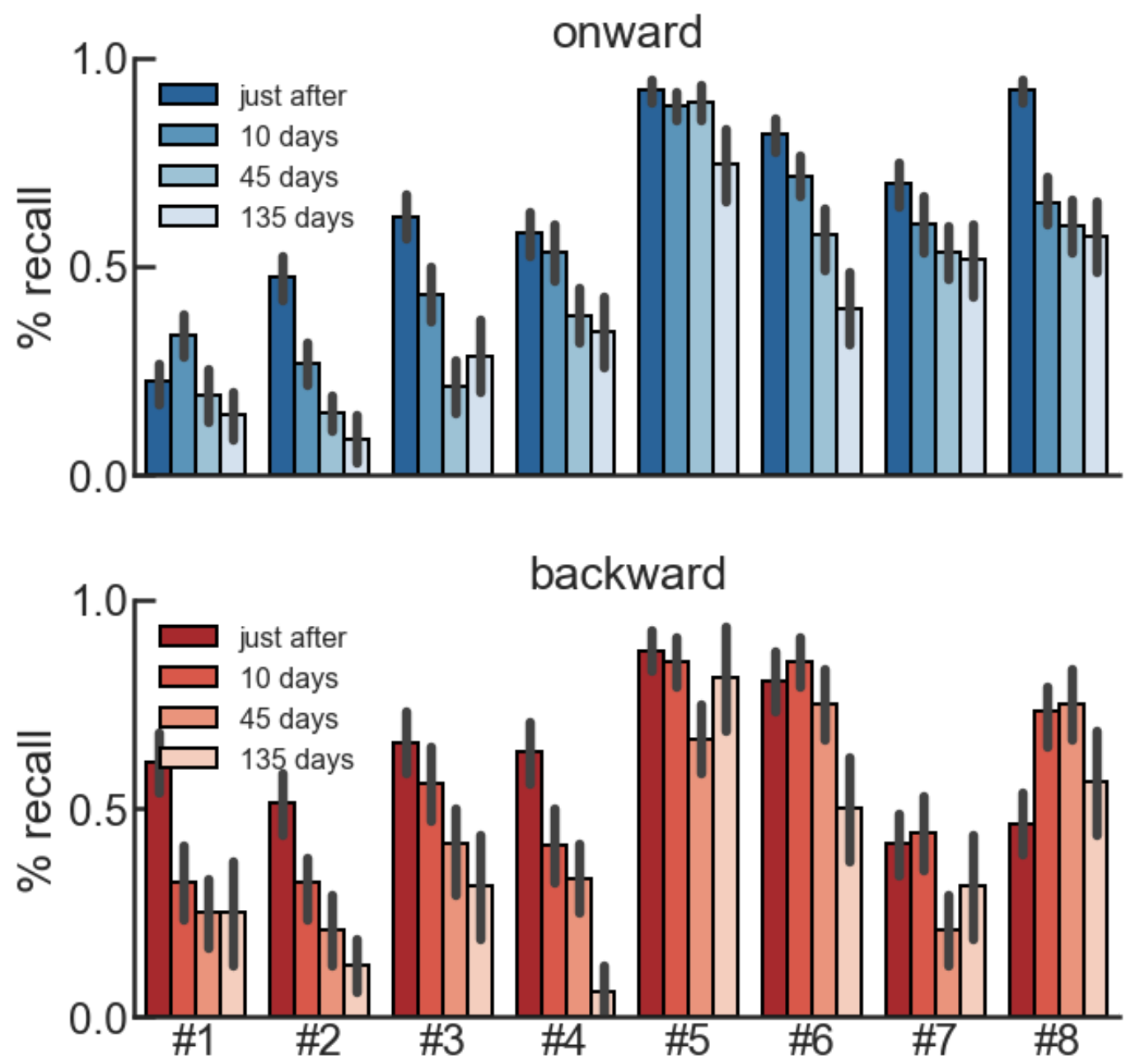

Supl. 3. Percentage of recall per trick in onward/backward. Onward graph was obtained by collapsing the results from onward\#1 and onward\#2. Backward is the same as in Supl. 1. We modeled the recall of each trick as a function of time*order. The only tricks that presented significant interaction were trick\#1, trick\#4 and trick\#8 (Fig. 4 and Supl. 8). 


\begin{tabular}{|c|c|c|c|c|}
\hline \multicolumn{5}{|c|}{ Generalized linear mixed model } \\
\hline \multicolumn{5}{|c|}{ Family: binomial (logit) } \\
\hline \multicolumn{5}{|c|}{ Formula: recall trick\#1 order*time+ (1 | subject) } \\
\hline AIC & BIC & LogLik & deviance & df.resid \\
\hline 348,6 & 367,7 & $-169,3$ & 338,6 & 330 \\
\hline \multicolumn{5}{|c|}{ Random effects } \\
\hline Group & Variance & Std. Deviation & n. groups & n. observations \\
\hline subject & 5,85 & 2,418 & 117 & 335 \\
\hline \multicolumn{5}{|l|}{ Fixed effects } \\
\hline & Estimate & Std. Error & $z$ value & p \\
\hline intercept & $-1,548$ & 0,6 & $-2,578$ & $0,0099 * *$ \\
\hline order & 3,38 & 1,032 & 3,277 & $0,001 * *$ \\
\hline time & $-0,241$ & 0,21 & $-1,144$ & 0,253 \\
\hline order*time & $-1,069$ & 0,402 & $-2,659$ & $0,008 * *$ \\
\hline
\end{tabular}

Supl. 4. Model for recall of trick\#1. Significant interaction sequence order with time. Trick\#1 is more recalled in the backward sequence, as it is presented as the last one. The difference just after the show vanishes with time, leading to a significant interaction.

\section{Generalized linear mixed model}

Family: binomial (logit)

Formula: recall trick\#8 order*time+ (1 | subject)

\begin{tabular}{|c|c|c|c|c|}
\hline AIC & BIC & LogLik & deviance & df.resid \\
\hline 370,3 & 389,4 & $-180,2$ & 360,3 & 330 \\
\hline Random effects
\end{tabular}

\begin{tabular}{|c|c|c|c|c|}
\hline Group & Variance & Std. Deviation & n. groups & n. observations \\
\hline subject & 3,751 & 1,937 & 117 & 335 \\
\hline \multicolumn{5}{|l|}{ Fixed effects } \\
\hline & Estimate & Std. Error & $z$ value & p \\
\hline intercept & 3,988 & 0,727 & 5,488 & $4,06 \times 10-8 * * *$ \\
\hline order & $-3,91$ & 0,948 & $-4,118$ & $3,83 \times 10-5 * * *$ \\
\hline time & $-1,07$ & 0,235 & $-4,538$ & $5,69 \times 10-6 * * *$ \\
\hline order*time & 1,267 & 0,333 & 3,806 & 0,00014 \\
\hline
\end{tabular}

Supl. 5. Model for recall of trick\#8. Significant interaction sequence order with time. Trick\#8 is more recalled in the onward sequence, as it is presented as the last one. The difference just after the show vanishes with time, leading to a significant interaction. 

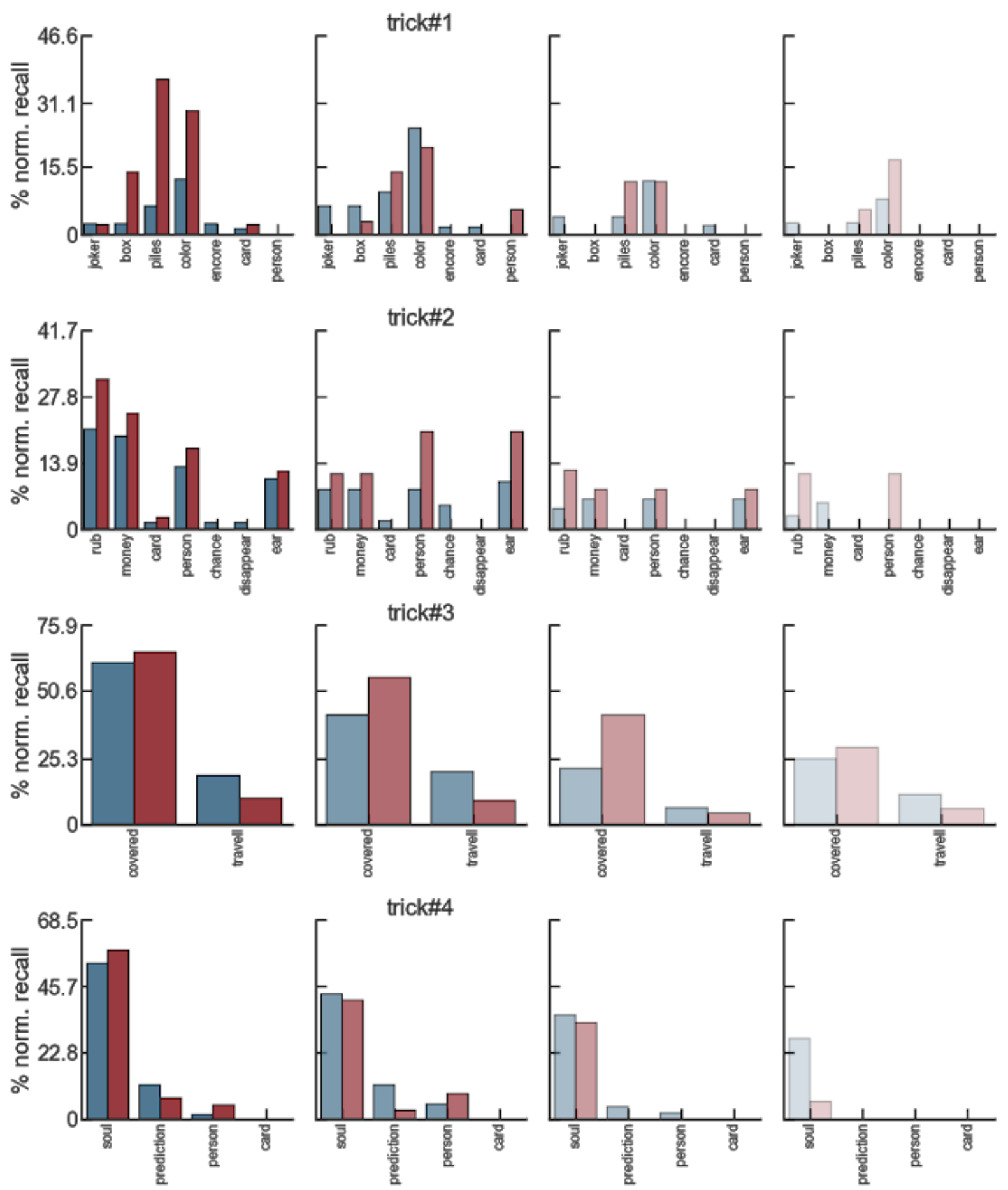

Supl. 6. Analysis of saliences of trick\#1 to trick\#4. Recall of the saliences in each trick. The recall is normalized by the total amount of saliences per trick. Regarding trick\#1, three saliences presented significant differences between the onward and background sessions, with higher values for the backward session (box: $t=-2.45, p=0.014$, piles: $t=-4.11, p<0.001$, color: $t=$ $-2.13, p=0.033$ ). Critically, these differences were only present after the trick (left), and not from 10 days up to 135 days after (center and right). 

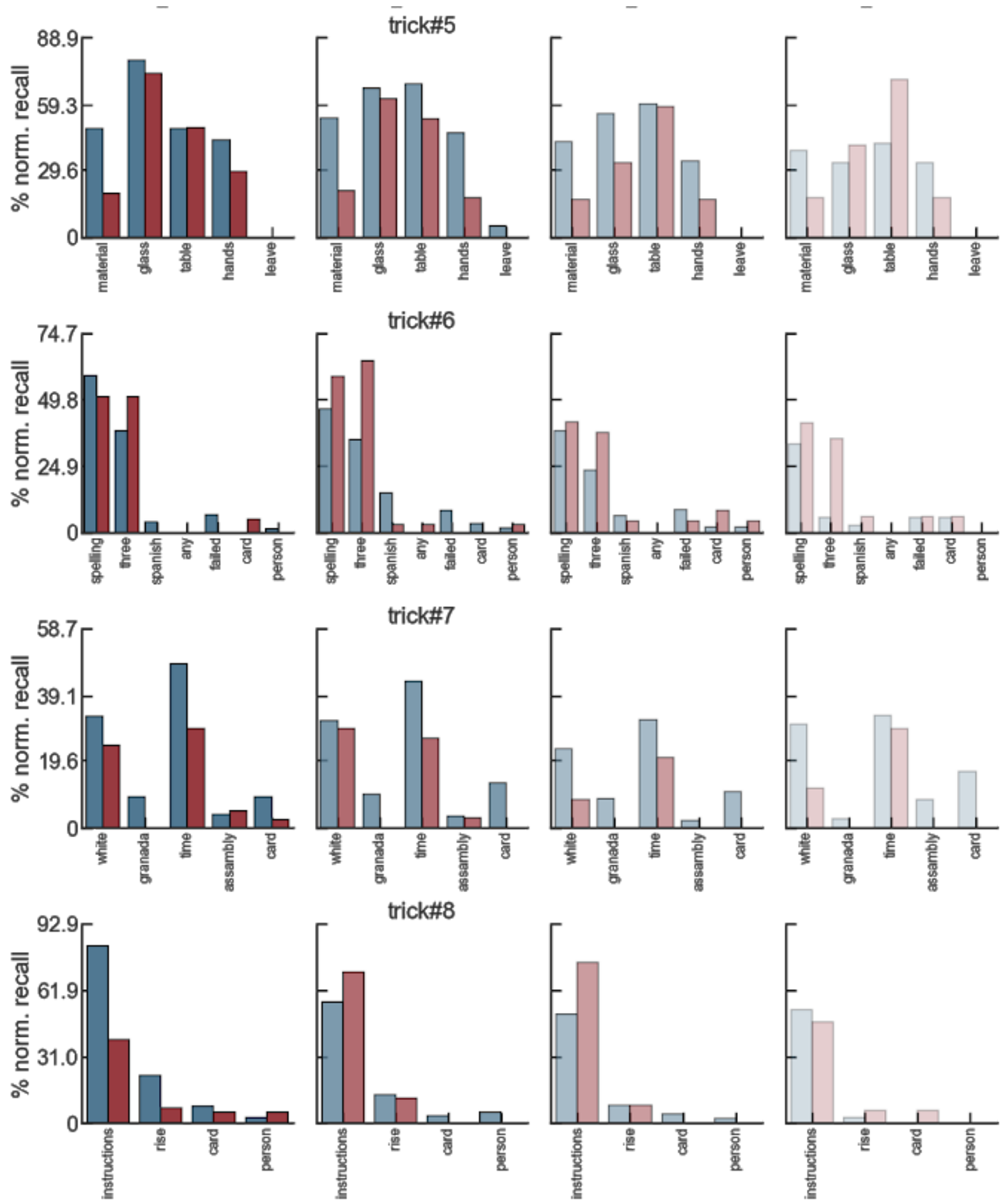

Supl. 7. Analysis of saliences of trick\#5 to trick\#8. Saliences recall in each trick. The recall is normalized by the total amount of saliences per trick. Regarding trick\#5, the salience material presented significant difference between the onward and the backward sessions in all first three times ( $t 1: t=3.1, p=0.002, t 2: t=3.1, p=0.002, t 3: t=2.2, p=0.029$ ) but not in the last recall. In trick\#8, two silences presented a significant difference between the onward and the backward session, just in $\mathrm{t} 1$ (instructions: $\mathrm{t}=4.83, \mathrm{p}<0.001$ and rise: $\mathrm{t}=2.063, \mathrm{p}=0.039$ ). Critically, higher recall was observed for onward sessions. 

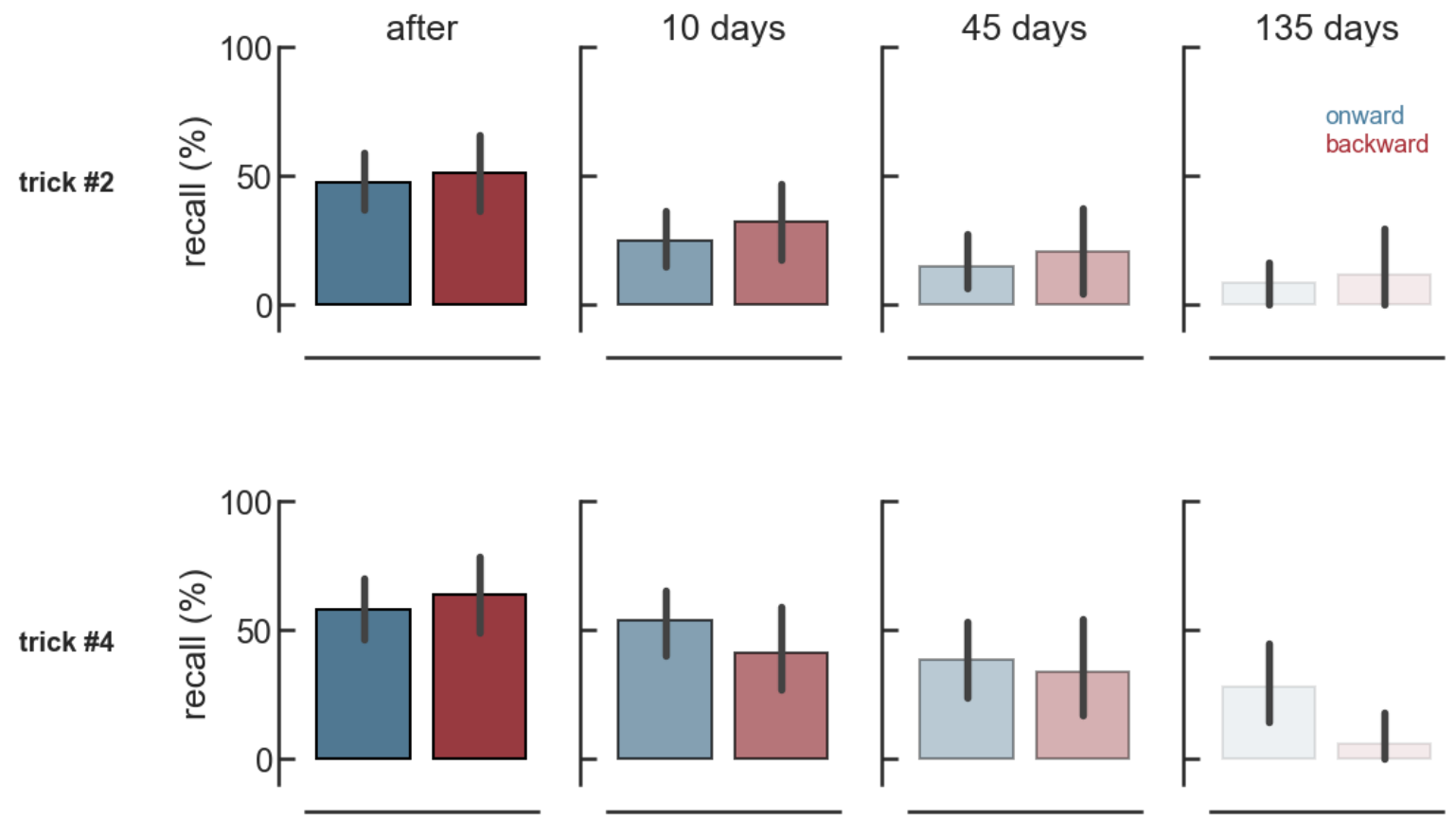

Supl. 8. Tricks extinguished after $\mathbf{4 . 5}$ months. In trick\#2, the memory is extinguished after 4.5 months for both the onward and backward session. In these plots, the error bars is a $95 \% \mathrm{Cl}$ to illustrate there is no difference with 0 (in the rest of the plots the $\mathrm{Cl}$ is the s.e.m). In trick\#4, this extinction occurs just in the backward session. Therefore, the interaction order-time emerges ( $\beta=-1.07, \mathrm{SE}=0.46, \mathrm{t}=-2.3, \mathrm{p}=0.02$ ). 